\title{
Stage IIB Bone Sarcoma AJCC v7
}

National Cancer Institute

\section{Source}

National Cancer Institute. Stage IIB Bone Sarcoma A/CC v7. NCI Thesaurus. Code C6466.

Stage IIB includes: T2, NO, M0, G3, G4. T2: Tumor more than $8 \mathrm{~cm}$ in greatest

dimension. N0: No regional lymph node metastasis. M0: No distant metastasis. G3:

Poorly differentiated. G4: Undifferentiated. (AJCC 7th ed.) 